\title{
Indoor mould growth prediction using coupled computational fluid dynamics and mould growth model
}

\author{
Majeed Olaide OLADOKUN ${ }^{1, *}$, Maisarah $\mathrm{ALI}^{2}$, Samsul Bahrin OSMAN ${ }^{3}$, Zhang LIN ${ }^{4}$
}

\author{
${ }^{1}$ Department of Architectural and Civil Engineering, City University of Hong Kong, Hong \\ Kong S.A.R. \\ ${ }^{2}$ Department of Civil Engineering, International Islamic University, Malaysia (IIUM), P. O. \\ Box 10, 50728, Gombak, Kuala Lumpur, Malaysia. \\ ${ }^{3}$ Department of Manufacturing and Material Engineering, International Islamic University \\ Malaysia (IIUM), Gombak Kuala Lumpur, Malaysia. \\ ${ }^{4}$ Division of Building Science and Technology, City University of Hong Kong, Hong Kong \\ S.A.R.
}

*Corresponding author: lyday011@yahoo.com, moladokun2-c@my.cityu.edu.hk

\section{(Blinded Manuscript)}

\begin{abstract}
This study investigates, using in-situ and numerical simulation experiments, airflow and hygrothermal distribution in a mechanically ventilated academic research facility with known cases of microbial proliferations. Microclimate parameters were obtained from in-situ experiments and used as boundary conditions and validation of the numerical experiments with a commercial computational fluid dynamics (CFD) analysis tool using the standard $k-\varepsilon$ model. Good agreements were obtained with less than $10 \%$ deviations between the measured and simulated results. Subsequent upon successful validation, the model was used to investigate hygrothermal and airflow profile within the shelves holding stored components in the facility. The predicted in-shelf hygrothermal profile was superimposed on mould growth limiting curve earlier documented in the literature. Results revealed the growth of xerophilic species in most parts of the shelves. The mould growth prediction was found in correlation with the microbial investigation in the case-studied room reported by the authors elsewhere. Satisfactory
\end{abstract}




\section{Building Simulation \\ Volume 10, pages 551-562(2017)}

prediction of mould growth in the room successfully proved that the CFD simulation can be used to investigate the conditions that lead to microbial growth in the indoor environment.

Keywords: Hygrothermal performance; in-situ experiments; microbial growth prediction; CFD simulation; indoor microclimate.

\section{Introduction}

\subsection{Building energy efficiency and indoor air quality}

With increased universal concern on urbanisation, rising energy cost, depletion of fossil fuels and greenhouse gas emission, the need for building energy efficiency remains a great concern to government and private agencies globally. The building sector becomes more of concern as its energy usage stands at about $40 \%$ of the global utilisation and contributing up to nearly 40$50 \%$ of the world carbon emissions (Hajdukiewicz et al. 2013, Calautit and Hughes 2014). Narrowing down the sector's energy utilisation by end-use revealed that the Heating, Ventilating and Air Conditioning (HVAC) systems take a lead with over $60 \%$ of the total building energy consumption (Wang et al. 2014). Building HVAC systems are tasked with provision of adequate thermal comfort as well as removal of contaminants and other indoor pollutants (Jelena 2011, Zhong et al. 2012). As a consequent, inadequate indoor ventilation will results in not only thermal discomfort but also unsatisfactory indoor air quality.

Unsatisfactory indoor air quality leads to Sick Building Syndrome (SBS) and other Building Related Illnesses (BRI). Similarly, poor ventilation is detrimental to building fabrics and indoor stored components of archives in the museums, library and other mission-critical indoor environments. In health care facilities, the ventilation system is tasked with preventing cross infection while maintaining adequate thermal comfort to the patients, the caregivers and visitors. On the contrary, despite the high energy consumption, the HVAC contributes to making building unhealthy (Wilson et al. 2007). In addition, evidence exists of an association 


\section{Building Simulation \\ Volume 10, pages 551-562(2017)}

between building energy efficiency improvements and elevated moisture level, microbial infestation as well as other deteriorative occurrences (Hens 1992, Di Giuseppe 2013). Therefore, there is need to consider hygrothermal performance of building when implementing energy efficiency and/or conservation measures. The built environment, over the past few decades, has therefore witnessed the emergence of building performance diagnostics.

\subsection{Building performance diagnostics}

Building performance assessments are executed on the existing buildings in retrofit upgrades as well as new ones even before they are built. According to Chen (2009), building performance appraisal are executed by: analytical and empirical methods, numerical simulation and experimental measurements. While experimental approach is found most significant as it generates validation data for analytical and numerical simulation models, field experiments are expensive in terms of cost and access to free houses (Essah et al. 2009).

Various performance metrics exist for assessing indoor air distribution based on the system's tasks (contaminant removal, air exchange, heat removal, etc.) and occupant's protection (Cao et al. 2014). In hygrothermal performance assessment, the thermohygric parameters are treated as contaminant and therefore can be investigated with contaminant removal related metrics (e.g. local mean age (LMA) of air). In an ideal indoor space with high airflow, the rate of air movement over the surfaces is higher thus maintain the surfaces at equal hygrothermal profile. In essence, high airflow supports higher movement of water molecules thereby reducing the rate of moisture deposition on the surfaces. This underscores elevated hygric profile and associated mould growth with inadequate ventilation performance (Ali et al. 2014). Such risks is higher in mechanical than naturally ventilated buildings as was previously reported by Borrego and Perdomo (2011) where it was found that fungal contamination was reduced in naturally than the mechanically ventilated buildings. The reason, according to the study, is due to the natural ventilation that facilitate the movement of particles in the air thereby 


\section{Building Simulation \\ Volume 10, pages 551-562(2017)}

making spore deposition difficult. Therefore, with the proven ability of LMA as a ventilation performance metrics for air freshness, its application to investigating indoor thermohygric balance and microbial contamination becomes promising in building performance assessments.

The rapid improvement in computer power in the past two to three decades has significantly influenced the development in computational models and progress in fluid dynamics research (Nielsen et al. 2007). Accordingly, there is a change in speed from about $10^{9}$ Flops in 1984 to $10^{13}$ Flops in 2002, an evidence that is corroborated by the observation of Li and Nielsen (2011). Various computational tools have therefore been developed as reviewed by Woloszyn and Rode (2008) and Delgado et al. (2013). These tools are grouped into building energy simulation (BES), heat air and moisture (HAM) and computational fluid dynamics (CFD) models with each of them having their strengths and weaknesses. Hence, the performance assessment can be accomplished within a single tool or by coupling several tools for improvement in their prediction of both energy performance, moisture performance and indoor environment quality analysis (Clarke 2013). Coupling between building performance tools can be done within a single program suite or by external data exchange between varying tools (Nielsen et al. 2007).

Despite these advancements, numerous uncertainties exist in building performance assessments. With good uncertainty clarifications, interpretation of results can be correctly done thereby increasing the reliability of obtained results. Experimental errors or uncertainties can arise from the set-up, data collection and measuring equipment (Jelena 2011). Similarly, uncertainties exists due to modelling simplifications, mathematical and numerical models, computational grids, and boundary conditions (Nielsen et al. 2007). Standard procedures on the execution and reporting of numerical experiments have evolved to reduce such uncertainties (Chen and Srebric 2002, ASHRAE 2013). Benchmarking selected code with a previously documented numerical and experimental solution (verification) is recommended for 


\section{Building Simulation \\ Volume 10, pages 551-562(2017)}

ascertaining uncertainty from the simulation code while correlating simulated results with the measured values (validation) to ensuring that the results obtained from simulation are less prone to errors. For errors and uncertainties due to computational grids, grid dependency analysis remains the industry standards for improving discretisation accuracy.

\subsection{Mould and mould growth prediction}

Moulds are pervasive in nature as they are primary decomposer of organic materials from plants and animal remains. As a results of mould ubiquity, they are omnipresent in indoor environment as microbiologically clean buildings do not exist (Elumalai et al. 2014). Despite their ecological importance, mould may be harmful to not only the indoor stored collections but also the occupants' health (Steeman et al. 2010). Mould limiting growth factors are numerous ranging from spores, sufficient temperature, nutrients and moisture. Mould does not require light to grow (JKR 2009) and as such believe on light as a growth factor appears erroneous (Clarke 2001). In predicting mould occurrence, moisture occurrence remains a good indicator of growth as mould proliferation is indicative of excessive moisture (BSI 2012). In addition, the length of time when the conditions prevail is also critical to mould growth. Mould growth is absent when the duration of elevated hygric conditions (RH greater than $80 \%$ ) is below three hours daily but when the same hygric condition prevails for over six hours, there is likelihood of mould growth (CIBSE 2015). Therefore a holistic approach is needed to predict where, when and under what conditions mould grows as early detection is often difficult until growth has advanced (Clarke 2013).

There are various models for mould growth prediction (Vereecken and Roels 2012) with no widely accepted evaluation method exists (Hagentoft and Sasic Kalagasidis 2010). Mould prediction models employ moisture presence (liquid or vapour form) with the humid air attaining a value above certain threshold limits. The concept of mould growth potential, $m$, using predicting equation was presented in the study of Hagentoft and Sasic Kalagasidis 


\section{Building Simulation \\ Volume 10, pages 551-562(2017)}

(2010). Accordingly, $m$ is defined as the relative humidity divided by the critical relative humidity for mould growth to start. The study submitted that mould growth will occur when $m$ exceeds unity $(m>1)$. This critical humidity is also defined as water activity, $a_{w}$ (ASHRAE 2009) with values above 0.8 sufficient enough for mould germination and growth. With the water activity of $0.8(\mathrm{RH}=80 \%)$, the indoor air ambient has to be within an hygric potential of $70 \%$ due to an existence of $10 \%$ increase between the ambient humidity and the surface moisture level (BSI 2007). Desjarlais et al. (2001) develops mould index with classifications on a scale of 0 to 6 , where 0 means no mould growth and 6 depicts deep growth that is capable of visual detection. In addition, mould growth limiting curve (Figure 1) was presented through an extensive study by Clarke and his research group (Clarke et al. 1997, Rowan et al. 1997, Clarke 2001). The limiting curves shown in Figure 1 represents the least combination of local surface temperature and humidity that support growth on surfaces of building elements and stored components. Mould growth is sustained as long as such hygrothermal conditions exist but ceases below any of the curves resulting in no growth for mould in that category. The mould categorisation provides six groups ranging between xerophilic (dry-loving) and hydrophilic (wet loving).

Mould growth index and mould growth limiting curve are good prediction tools. The distinction in their usage arise from the fact that while growth index consider growth visibility, the growth limiting curve predicts the likely species that grows under a given indoor temperature and relative humidity. It therefore appears mould growth index is more suitable for visual inspection while growth limiting curve lends itself towards predicting the probable species under given operative conditions. This capability of mould growth limiting curve place it in a position of suitability for coupling with building performance simulation tools where the prevailing operative conditions can be modelled to predict mould growth. Such combined prediction with mould growth model and building performance simulation tool, especially for 
CFD, appears limited in the body of existing studies. The present study is, therefore, set to demonstrate the use of CFD in mould growth prediction. The method is applied to a mechanically ventilated academic research facility with known cases of microbial proliferations using in-situ experiment and CFD simulation coupled with mould (limiting curve) prediction model. A similar study with building energy simulation tool, ESP-r, was carried out and reported in the work of Clarke (2013).

\section{Materials and Methods}

\subsection{In-Situ experiments}

\subsubsection{Experimental setup}

The case-studied room, measures $5.2 \mathrm{~m}$ long by $4.8 \mathrm{~m}$ wide and $3.0 \mathrm{~m}$ high. It is air-conditioned and ventilated by a constant air volume (CAV) air handling unit (AHU) that controls the airflow, thermal and hygric distribution in the room. The air distribution is a mixing ventilation type with a ceiling-mounted four-way square supply diffuser $(600 \mathrm{~mm} \times 600 \mathrm{~mm})$ and a rectangular extract grille $(600 \mathrm{~mm} \times 300 \mathrm{~mm})$. The lighting system consists of six numbers ceiling-mounted fluorescent fittings $(600 \mathrm{~mm} \times 1200 \mathrm{~mm})$ with two numbers $36 \mathrm{~W}$ lamps. The air outlets as well as light fittings were flushed with the ceiling surface. The furniture comprises of metal shelves to keep stored components. Figure 2 shows the layout and measurement positions in the in-situ experimental setup.

\subsubsection{Instrumentation and measurement}

The in-situ experiments, which involved microclimate investigations, were in two manifolds: steady state and time-series. The steady state experiment involves collection of data that include (1) room surface temperature from wall, floor and ceiling (with TESTO infra-red thermometer); (2) room ambient air temperature, relative humidity and speed (with ALNOR AVM440 anemometers); and (3) supply and exhaust temperature, relative humidity and air 


\section{Building Simulation \\ Volume 10, pages 551-562(2017)}

flow rate (with ALNOR AVM440 anemometers). In the time-series measurement, data loggers (EL-USB-2-LCD+) were mounted at the geometrical room center, supply and exhaust outlets. In the steady state experiment, to measure air supply and return conditions, the face of supply diffusers and exhaust grilles were divided into grids to facilitate measurement of airflow, thermal and hygric parameters. Nine measurements were taken from each supply diffusers while six were taken for the exhaust grilles. The measurements were repeated in triplicates and averaged to give a fair representation of the measured parameters. As for the time-series data, the measurement was carried out for a period of six days with EL-USB-2LCD+ Data Logger that were configured to log data at every five minutes intervals. This resulted in 12 time-steps hourly data to give a total of 1728 (i.e. 12data-points x 24hours/day x 6days) matrix of hygrothermal parameters. Figure 3 shows the time-series data of hygrothermal profile in the case-studied room over the test period. As shown in the figure, the supply conditions in the room over the test period ranges between $13.0^{\circ} \mathrm{C}$ to $16.0^{\circ} \mathrm{C}$ temperature and $85.0 \%$ to $92.0 \%$ relative humidity. The elevated hygrothermal conditions in the test room and other related areas together with its implication on microbial growths had been reported elsewhere (Ali et al. 2014). Data from the steady state measurements were use as input boundary conditions for the CFD simulations while those from time-series measurement were used for boundary condition as well as model validation. Table 1 shows the specifications, accuracies and precisions of the measuring equipment.

As results of field measurements are susceptible to experimental uncertainties, the measured data are evaluated for uncertainty assessment using the methodology described in a previous study by one of the authors (Oladokun 2015). An acceptable level of uncertainty in the results of a measured variable is first determined as the level of confidence (LOC). The commonly applied LOC (Vincent and Weir 2012) of 95\% ( $\mathrm{p}<0.05)$ is we adopted in this study. To evaluate the measurement uncertainty, we applied Equation (1) for the confidence interval. 
$C I= \pm 1.96 \times S E_{M}$

Where $C I$ is the confidence interval and $S E_{M}$ is the standard error of the mean. The $S E_{M}$ is obtained from Equation (2) as follows:

$$
S E_{M}=\frac{\sigma}{\sqrt{n}}
$$

Where $\sigma$ is the standard deviation and $n$ is the number of observed time-series data. The $C I$ in equation (1) is an interval, hence the positive "+" and negative "-" signs in the equation. The calculated $C I$ value is compared with the equipment accuracy provided by the manufacturer. The values of $C I$ below or at most equal to the equipment accuracy indicates certainty in the obtained parameters at $95 \%$ LOC (Oladokun 2015). The author further submitted that using this approach, where variation occurs in measurement at various locations, such variations can be attributed to causes other than the measuring equipment. On the other hand, when $C I$ exceeds the equipment accuracy, equipment error is suspected and may suggest need to calibrate such measuring equipment.

\subsection{Numerical simulation}

\subsubsection{Governing equation}

In the numerical simulations, incompressible steady state Navier-Stokes equation coupled with the standard $k-\varepsilon$ turbulence model were employed. General form of the Navier-Stokes equation (Nielsen et al. 2007) is shown in Equation (3). Table 2 presents the dependent variables $(\phi)$, effective diffusion coefficients $(\Gamma \phi)$, and the source term $(S \phi)$ for each of the flow parameters in Equation (1). 


$$
\begin{gathered}
\text { Building Simulation } \\
\text { Volume 10, pages 551-562(2017) } \\
\frac{\partial}{\partial t}(\rho \varphi)+\operatorname{div}\left(\rho V \varphi-\Gamma_{\varphi} \operatorname{grad} \varphi\right)=S_{\varphi}
\end{gathered}
$$

Where $\rho$ is the density, $V$ is the velocity vector, $\phi$ is the dependent variable in the flow field to which the equation applies (temperature, velocity, pressure, etc.), $\Gamma \phi$ is the turbulent diffusion coefficient and $S \phi$ is the source or sink term of the variable $\phi$.

\subsubsection{Benchmark case}

In numerical experiments, it is essential for users of a specific tool to reproduce an existing study with reported experimental and numerical data (Jelena 2011). This is to ascertain the capability of the selected tool to carry out the experiment. In addition, the users' ability of CFD code requires verification alongside the code performance. Such verification procedures are termed benchmarking. It should be noted that the term benchmarking is also used in building performance diagnostic where two similar models (physical or computational) are compared for performance assessments. In this study, the term benchmark is employed to indicate verification of both the CFD tool's capability as well as the simulator's ability to solve problems involving airflow and mass (contaminant and heat) transport in indoor environment.

As a result and owing to the documented procedures (Chen and Srebric 2002, Srebric and Chen 2002, ASHRAE 2013), the present study carried out a benchmark case using results from Spitler (1990) experiments. The benchmark is aimed at: (1) ascertaining the capability of the selected CFD code to simulate and reproduce the selected physical phenomena as previously documented with minimal deviations, and (2) to equip the researcher with necessary experience in simulating indoor air related problems together with making an informed judgment from such simulation results. The original study measures and compares temperature, airflow and other parameters along the room height, exhaust outlet and indoor ambient. This study, therefore, replicates the Spitler experiment using CFD with temperature and airflow 
measurement along the room height selected as benchmarking parameters. Basic boundary conditions in the benchmark case are - Room size: $4.57 \mathrm{~m} \times 2.74 \mathrm{~m} \times 2.74 \mathrm{~m}$ high; air supply and exhaust outlets: size $=903.7 \mathrm{~mm} \times 400 \mathrm{~mm}$, temperature $=21^{\circ} \mathrm{C}$, airflow rates $=15 \mathrm{ACH}$; heated panels on all wall surfaces with temperature $=30^{\circ} \mathrm{C}$.

\subsubsection{Experimental model setup}

The 3D modelling (Figure 4) of the experimental case-studied room was created within the CFD tool. In the model, the shelves were placed nearly $25 \mathrm{~mm}$ from the wall surfaces and set directly on the floor. The stored items were modelled as a row of objects rather than individual items to conserve computational resources. In addition, since the objects allow air movement between them, they were modelled as resistances (porous object) with $20 \%$ openings. The simplification is considered sufficient to represent the airflow through the objects as similarly adopted by Fletcher et al. (2001). One of the many benefits of CFD simulation is whole-field visualization thereby supporting placement of virtual data loggers at different points of interests other than the in-situ measurement positions. The use of virtual data loggers (in this study) allow assessment of the hygrothermal conditions within the shelves and stored items that seems difficult to achieve on site. Figure 2 shows details of the space layouts with positions of supply, exhaust and other components of the case-studied room.

\subsubsection{Boundary condition, discretisation and grid dependency analysis}

The source terms in Equation 1 are provided as boundary conditions from the air outlets supply diffuser (temperature $=15.5^{\circ} \mathrm{C}$, humidity ratio $=9.8 \times 10^{-3} \mathrm{~kg} / \mathrm{kg}$, airflow rates $=0.61$ $\mathrm{m}^{3} / \mathrm{s}$ ) and return grille (airflow rates $=0.61 \mathrm{~m}^{3} / \mathrm{s}$ ). Others are enclosure surface temperatures walls $\left(17.4^{\circ} \mathrm{C}, 16.8^{\circ} \mathrm{C}, 18.4^{\circ} \mathrm{C}\right.$ and $\left.19.6^{\circ} \mathrm{C}\right)$, floor $\left(18.4^{\circ} \mathrm{C}\right)$ and ceiling $\left(17.3^{\circ} \mathrm{C}\right)$ and lighting heat gains $(6 \times 72 \mathrm{~W})$. The return grille humidity ratio as well as temperature are to be computed by the CFD simulation. As the accuracy of CFD results depends on the grids' quality 


\section{Building Simulation \\ Volume 10, pages 551-562(2017)}

(Chen and Srebric 2002, Nielsen et al. 2007), the entire simulation domain was discretised using structured grid approach. Grid refinement was performed around the supply inlets and return outlets to cater for high gradients often associated with air terminal devices thereby improving the prediction of velocity flow field (Calautit and Hughes 2014). The baseline grid was of coarse type with a total 4752 cells. Grids fine-tuning was executed with a total of 9885 and 39935 for medium and fine grids respectively. In this study, grid dependency analysis was carried out with virtual measurement of the hygrothermal parameters on a vertical section at center of the modelled room. Series of simulation were executed using coarse, medium and fine grids.

\subsubsection{Model validation}

Subsequent upon the satisfactory grid selection, the model validation is executed between the measured and simulated hygrothermal parameters in the room center. In the validation, the study adopted percentage root-mean square deviation (PRMSD) approach (Lim et al. 2013). PRMSD is obtained as a single value for the reference points as evaluated from Equation (2):

$$
P R M S D=\sqrt{\frac{1}{n} \sum_{1=0}^{n}\left(\frac{c_{m}-c_{s}}{c_{m}} \times 100\right)^{2}}
$$

Where: $C_{m}$ is the measured parameter; $C_{s}$ is the simulated parameters and $n$ is the number of points under considerations.

\subsubsection{Simulation of hygrothermal profile on stored items and mould growth prediction}

Extended simulations were carried out on the model in which key parameters were selected for further analysis to predict the hygrothermal parameters ( $\mathrm{T}$ and $\mathrm{RH}$ ) within the shelves and racks. Also, the average LMA in the room is selected as a baseline and plotted on graph of 


\section{Building Simulation \\ Volume 10, pages 551-562(2017)}

hygrothermal and air flow profiles. Selected points along the $x$-axis were chosen to assess the indoor thermohygric and LMA distributions at planes before $(0.75 \mathrm{~m})$ and after $(2.25 \mathrm{~m})$ the supply diffuser. In order to predict the likely species of mould likely to grow under the prevailing operative conditions in the case-studied room, the simulated in-shelves hygrothermal conditions were coupled with the mould growth limiting curve (otherwise termed as isopleth). Results of the predicted hygrothermal profiles were superimposed on the mould growth isopleth of Clarke et al. (1996). A similar approach had been previously reported in Clarke (2013) where BES code (ESP-r) was used to predict the hygrothermal conditions leading to growth in a mould infested house.

\section{Results and Discussions}

\subsection{Measurement uncertainty analysis}

Table 3 compares the results of uncertainty assessment from the measuring tools with the manufacturer's specifications. As shown in the table, the measurement uncertainty for temperature approximately ranges between \pm 0.2 to \pm 0.4 while that of humidity varies between $\pm 0.6 \%$ and $\pm 0.7 \%$. Overall, the estimated equipment uncertainty falls below the manufacturer's specified accuracy for the sensors. It is, therefore, sufficient to conclude with 95\% confidence that the measurements are accurate and variations between measurement points will be due to reasons other than those related to the measuring equipment uncertainties. Nevertheless, there is a $5 \%$ chance that the results are inaccurate, the value that is statistically acceptable.

\subsection{Benchmark case}

This section describes the detail comparison between Spitler experiment and the verification studies. Figure 5 shows the airflow contour plots measured at plane cutting across the centre of the supply outlet. The airflow contour plots revealed similar flow pattern between the CFD 


\section{Building Simulation \\ Volume 10, pages 551-562(2017)}

simulated (Figure 5b) and the Spitler experiment (Figure 5a). The cold air drops from the supply outlet and flow parallel to the floor until it gets to the opposite wall. Subsequently, the air rises along the wall and when it gets to ceiling level, the air vortexes out. Overall, the airflow contour reveals a very good agreement between the simulated and Spitler results. Results of the percentage root-mean square deviation (PRMSD) between the CFD simulation and experimental thermal data of Spitler revealed a maximum value of $7 \%$. This deviation falls within the documented acceptable limits (Kavgic et al. 2008, Gong et al. 2011, Lim et al. 2013, Calautit and Hughes 2014). The result therefore indicates that both the CFD simulation code and the investigator were able to produce results of good agreement with a previously documented experimental data. Hence, it can be concluded that the researcher is capable of using CFD tool to satisfactorily model and simulate problems involving indoor airflow.

\subsection{Grid dependency analysis}

Figure 6 shows the results of grid dependency assessments. The prediction from coarse grid was far low in comparison to medium and fine grids. Nevertheless, for most of the measured cases, the fine mesh under-predicts the basic flow parameters as compared to medium grids. This reason, coupled with longer computational time resulted in selecting the medium grid for further analysis.

\subsection{Validation of simulation and measured data}

PRMSD between the measured and simulated parameters for the in-situ as well as simulated experiments gave $2.6 \%$ and $4.1 \%$ respectively for $\mathrm{T}$ and $\mathrm{RH}$. The validation results performed better than earlier studies that acceptable deviations between the measured and simulated values in numerical investigation should be in the range of 5\% (Gong et al. 2011) or 10\% (Calautit and Hughes 2014). In the study of (Kavgic et al. 2008), the difference was found to 


\section{Building Simulation \\ Volume 10, pages 551-562(2017)}

be higher than $5-10 \%$ where their experiment reported a deviation of more than $25 \%$. A value of $6.7 \%$ deviation was reported in the work of (Lim et al. 2013).

\subsection{Thermohygric and Local Mean Age profiles}

The mean thermal, hygric and air age performances in the occupied zone were found as $\mathrm{T}=$ $17.2{ }^{\circ} \mathrm{C}, \mathrm{RH}=78.4 \%$ and $\mathrm{LMA}=149.2 \mathrm{~s}$. Figures 7 and 8 show the thermohygric profiles at $x=0.75 \mathrm{~m}$ and $2.25 \mathrm{~m}$ respectively. The upper part close to ceiling shows high temperature and low humidity due to heat emission from lighting while concentrated cold region is shown on the air-stream around the supply diffuser (Figures 7a and 8a). In addition, regions close to the shelves were found with low thermal and elevated hygric gradients (Figures $7 \mathrm{~b}$ and $8 \mathrm{~b}$ ). It was revealed that thermal and hygric stratifications existed not only along the height in the room but also around the shelves.

Similar to the thermohygric stratifications, the LMA profile were shown with stratified layers in the room (Figures 9a and 9b). As LMA measures the rate of freshness of air, the locations with longer LMA appear stale and vice versa. Higher LMA is therefore synonymous with high contaminant loadings at such locations. In hygrothermal assessment, the contaminant is mainly the air water vapour, hence the risk of higher moisture deposition increases with LMA. Looking at the LMA profile reveals that longer LMA characterises the occupied zone as well as the shelf areas which suggest the reasons for higher risk of thermohygric imbalance. These findings suggest that the air in the room is not well distributed leading to hygrothermal stratifications. Consequence of such stratification in the thermal and high hygric profiles could result in microbial proliferation.

\subsection{Effect of LMA on thermohygric profile and mould growth}

Air staleness and contaminant loading increases with LMA. Since water vapour is considered as contaminant in hygrothermal assessment, high LMA results in higher risk of moist air 


\section{Building Simulation \\ Volume 10, pages 551-562(2017)}

deposit. Figures 10 and 11 show the results of in-shelf thermal, hygric and LMA profiles. The graphs also include the mean room LMA value of 149.2 seconds as baseline value for the room. It is found that most parts of the stacks have their LMA above the benchmark values. Shelve \#1 recorded the poorest performance (Figure 10) with eight out of nine points above 149.2 seconds. This presents shelve \#1 as having the highest risk of moisture build-up due to poor air movement. Coincidentally, shelve \#1 was found to have highest visible mould growth on the stored components (Figure 11). This proved that elevated LMA results in not only thermohygric imbalance but also mould growth risk.

Contrary to findings on shelve \#1, the results revealed lowest number of points within the stacks, on shelve \#3, with only three out of the nine locations operating at values above the benchmark LMA (Figure 12). This could be as a result of the shelves closeness to the supply and exhaust openings that facilitates easy air exchange. Shelves \#2 have five out of the nine points above the benchmark LMA (Figure 13), leaving the stack at a lower moisture build-up risk; hence reduced visible mould growth.

In general, the results of hygrothermal and LMA profiles revealed most part of the stacks with flow imbalance. This leads to staler air inside majority of the stacks thereby making moisture laden air to stay longer in most parts of the shelves. These stratifications could be primarily due the location of supply and exhaust openings which are located towards one side of the case-studied room. Optimisation could be made in this situation on the best locations for the supply diffuser and exhaust grille that will give a uniform distribution of air within and around the stacks. While that is acknowledged, such optimisation analysis is beyond the scope of the present studies and may therefore form part of our future studies.

\subsection{Mould growth prediction}

The predicted in-shelves hygrothermal conditions were superimposed on the mould growth isopleths. Figure 14 shows the predicted mould growth in the case-studied room. Each of the 


\section{Building Simulation \\ Volume 10, pages 551-562(2017)}

marked points represent stacks within the respective shelves. In most cases, the predicted mould groups were between categories A to $\mathrm{C}$ - the xerophilic (dry-loving) species of mould classifications (Clarke et al. 1996, Clarke 2001, Clarke 2013). Results of mould predictions were in agreement with the results of microbial sampling protocol reported elsewhere (Ali et al. 2014). It was found that the viable mould species in the room were mainly Penicillium sp. and Aspergillus sp.. These identified species were categorised as xerophilic (dry-loving) in the documented research of Clarke (1996, 2001). The findings from coupling CFD with mould growth prediction model correlate well with the microbial sampling. Hence, the methods presents in this study is promising in building performance diagnostics involving mould growth. It will therefore be of help to building surveyors and other IAQ inspectors.

\section{Conclusions}

Environmental sustainability in building requires energy efficient ventilation system that is often found with detrimental effects on the buildings and its users. Building performance diagnostic tools have developed over the past decades with the advancement in computational power and assessment metrics. Mould proliferations in indoor environment possess danger to occupants' health as well as the building and its stored components, hence early prediction becomes necessary. The study combines in-situ and numerical experiment with mould growth model to predict mould growth in a case studied building. Benchmark study in CFD simulation improves reliability on the analysis tool in its application of the governing equations in addition to proving the experimenter's ability. Similarly, validation of the simulated results with measured data provides certainty about the model implementation as well as the adopted procedures for the case consideration. Results of benchmark studies revealed a similarity between the documented and presently simulated results. This indicates that both the CFD analysis tool and the researchers were capable of providing solutions for indoor airflow performance assessments. The validation study provides a good agreement between the in-situ 
and numerical experiments with less than $10 \%$ deviation. Hence, the model is capable to represent the case-studied room. Thermal and hygric stratifications were found throughout most part of the room thereby suggesting that the air was not well distributed in the room, a situation that leads to microbial proliferations.

The results of mould growth prediction were found in correlation with microbial investigation in the case studied room. Xerophilic mould - Penicillium sp. and Aspergillus sp. were found in the case-studied room. Satisfactory prediction of mould growth in the room successfully proved that the CFD simulation can be used to investigate the conditions that lead to microbial growth in the indoor environment. It is a common practice in reality to optimise the design by running additional simulations. In the optimised simulation, various parameters can be varied and effects examined on the hygrothermal profile and mould growth likelihood. Such optimisation studies is beyond the scope of the present study and therefore recommended for further future investigations.

\section{Acknowledgements}

This work was supported by the Ministry of Higher Education (MOHE) Malaysia (FRGS12067-0126). The authors equally wish to acknowledge the assistance of Dr Niza Samsuddin, Dr Hairul Aini Hamzah as well as the Dean of Kulliyyah (Faculty) of Medicine IIUM together with the management and staffs of Daya Bersih during the studies.

\section{References}

Ali M, Oladokun MO, Osman SB, Samsuddin N, Hamzah HA, Salleh MN (2014). Ventilation Performance Assessment of an Educational Building in a Hot and Humid Climate. In: Proceedings of the InCIEC 2014: International Civil Engineering and Infrastructure Engineering Conference, pp. 333-344. 


\section{Building Simulation \\ Volume 10, pages 551-562(2017)}

ASHRAE (2009). ANSI/ASHRAE Standard 160-2009: Criteria for Moisture-Control Design Analysis in Buildings, American Society of Heating, Refrigerating and Air-Conditioning Engineers, Inc.

ASHRAE (2013). ASHRAE Guideline 33-2013: Guideline for documenting indoor airflow and contaminant transport modeling, American Society of Heating, Refrigerating and AirConditioning Engineers, Inc.

Borrego S, Perdomo I (2011). Aerobiological investigations inside repositories of the National Archive of the Republic of Cuba, Aerobiologia, 28: 303-316.

BSI (2007). BS EN 15026:2007 Hygrothermal performance of building components and building elements - Assessment of moisture transfer by numerical simulation, BSI Standards Limited.

BSI (2012). BS ISO 16000-19:2012 Indoor air — Part 19: Sampling strategy for moulds, BSI Standards Limited.

Calautit JK, Hughes BR (2014). Measurement and prediction of the indoor airflow in a room ventilated with a commercial wind tower, Energy and Buildings, 84: 367-377.

Cao G, Awbi H, Yao R, Fan Y, Sirén K, Kosonen R, Zhang J (2014). A review of the performance of different ventilation and airflow distribution systems in buildings, Building and Environment, 73: 171-186.

Chen Q (2009). Ventilation performance prediction for buildings: A method overview and recent applications, Building and Environment, 44: 848-858.

Chen Q, Srebric J (2002). A Procedure for Verification, Validation, and Reporting of Indoor Environment CFD Analyses, HVAC\&R Research, 8: 201-216.

CIBSE (2015) Moisture transfer and condensation, In: Butcher, K. (ed) CIBSE Guide A: Environmental design, London, The Chartered Institution of Building Services Engineers, 7-1 - 7-16. 
Clarke J (2013). Moisture flow modelling within the ESP-R integrated building performance simulation system, Journal of Building Performance Simulation, 6: 385-399.

Clarke JA (2001). Energy Simulation in Building Design, Oxford, Butterworth-Heinem.

Clarke JA, Johnstone CM, Kelly NJ, McLean RC (1996). Development of a technique for the prediction/alleviation of conditions leading to mould growth in houses, Final Report for Contract Number 68017, Scottish Homes.

Clarke JA, Johnstone CM, Kelly NJ, McLean RC, Nakhi AE (1997). Development of a Simulation Tool for Mould Growth Prediction in Buildings. In: Proceedings of the Proceedings of the Fifth International IBPSA Conference Vol. 2, pp. 343-349.

Delgado JM, Barreira E, Ramos NM, De Freitas VP (2013). Hygrothermal Numerical Simulation Tools Applied to Building Physics, Springer.

Desjarlais AO, Karagiozis AN, Aoki-Kramer M (2001). Wall moisture problems in Seattle, Buildings VIII proceedings, ASHRAE, $8 p$.

Di Giuseppe E (2013). Nearly Zero Energy Buildings and Proliferation of Microorganisms: A Current Issue for Highly Insulated and Airtight Building Envelopes, SpringerBriefs in Applied Sciences and Technology, eBook, Springer International Publishing.

Elumalai P, Elumalai E, David E (2014). Fungi Associated with Deteriorations of Painted Wall Surfaces: Isolation and Identification, European Journal of Academic Essays, 1: 48-50.

Essah EA, Sanders CH, Baker P, Kalagasidis AS (2009). Condensation and moisture transport in cold roofs: effects of roof underlay, Building Research \& Information, 37: 117-128.

Fletcher C, Mayer I, Eghlimi A, Wee K (2001). CFD as a Building Services Engineering Tool, International Journal on Architectural Science, 2: 67-82.

Gong G, Xu C, Jiao J, Liu Y, Xie S (2011). Investigation of moisture condensation on papermaking plant envelopes in high humidity environment by orthogonal analysis and CFD simulation, Building and Environment, 46: 1639-1648. 
Hagentoft C-E, Sasic Kalagasidis A (2010). Mold Growth Control in Cold Attics through Adaptive Ventilation. Validation by Field Measurements. In: Proceedings of the 12th International Conference on Performance of the Exterior Envelopes of Whole Buildings.

Hajdukiewicz M, Geron M, Keane MM (2013). Formal calibration methodology for CFD models of naturally ventilated indoor environments, Building and Environment, 59: 290302.

Hens H (1992). IEA Annex 14: Condensation and Energy, Journal of Building Physics, 15: 261-273.

Jelena S (2011) Ventilation performance prediction, In: Hensen, J. and Lamberts, R. (eds) Building performance simulation for design and operation, Taylor \& Francis.

JKR (2009). Guidelines on the prevention of mould growth in buildings, JKR Malaysia.

Kavgic M, Mumovic D, Stevanovic Z, Young A (2008). Analysis of thermal comfort and indoor air quality in a mechanically ventilated theatre, Energy and Buildings, 40: 13341343.

Li Y, Nielsen PV (2011). CFD and ventilation research, Indoor Air, 21: 442-453.

Lim CH, Saadatian O, Sopian K, Sulaiman MY, Mat S, Salleh E, Ng K (2013). Design configurations analysis of wind-induced natural ventilation tower in hot humid climate using computational fluid dynamics, International Journal of Low-Carbon Technologies, 0: 1-15.

Nielsen PV, Allard F, Awbi HB, Davidson L, Schälin A (2007). Computational Fluid Dynamics in Ventilation Design, In: Nielsen, P. V. (ed) REHVA Guidebook, Vol. 10, Finland, REHVA.

Oladokun MO (2015). Mould Growth Prediction in Tropical Climate Buildings by Hygrothermal Differentials. Msc Dissertation, International Islamic University Malaysia, Building Services Engineering. 
Rowan NJ, Anderson JG, Smith JE, Clarke JA, McLean RC, Kelly NJ, Johnstone CM (1997). Development of a Computer Programme for the Prediction and Control of Mould Growth in Buildings Using the ESP-r Modelling System, Indoor and Built Environment, 6: 4-11.

Spitler JD (1990). An experimental investigation of air flow and convective heat transfer in enclosures having large ventilative flow rates. PhD, University of Illinois at UrbanaChampaign.

Srebric J, Chen Q (2002). An example of verification, validation, and reporting of indoor environment CFD analyses (RP-1133), ASHRAE Transactions, 108: 185-194.

Steeman M, De Paepe M, Janssens A (2010). Impact of whole-building hygrothermal modelling on the assessment of indoor climate in a library building, Building and Environment, 45: 1641-1652.

Vereecken E, Roels S (2012). Review of mould prediction models and their influence on mould risk evaluation, Building and Environment, 51: 296-310.

Vincent W, Weir J (2012). Statistics in Kinesiology, Human Kinetics.

Wang Y, Fu-Yun Z, Kuckelkorn J, Liu D, Liu J, Jun-Liang Z (2014). Classroom energy efficiency and air environment with displacement natural ventilation in a passive public school building, Energy and Buildings, 70: 258-270.

Wilson SC, Palmatier RN, Andriychuk LA, Martin JM, Jumper CA, Holder HW, Straus DC (2007). Mold contamination and air handling units, Journal of Occupational and Environmental Hygiene, 4: 483-491.

Woloszyn M, Rode C (2008). Tools for performance simulation of heat, air and moisture conditions of whole buildings, Building Simulation, 1: 5-24.

Zhong K, Chen S, Kang Y, Yuan L (2012). Analysis of outdoor temperature ranges for the operation of different ventilating modes in commercial buildings of China, Energy and Buildings, 55: 174-182. 
Building Simulation

Volume 10, pages 551-562(2017) 


\section{List of Figures}

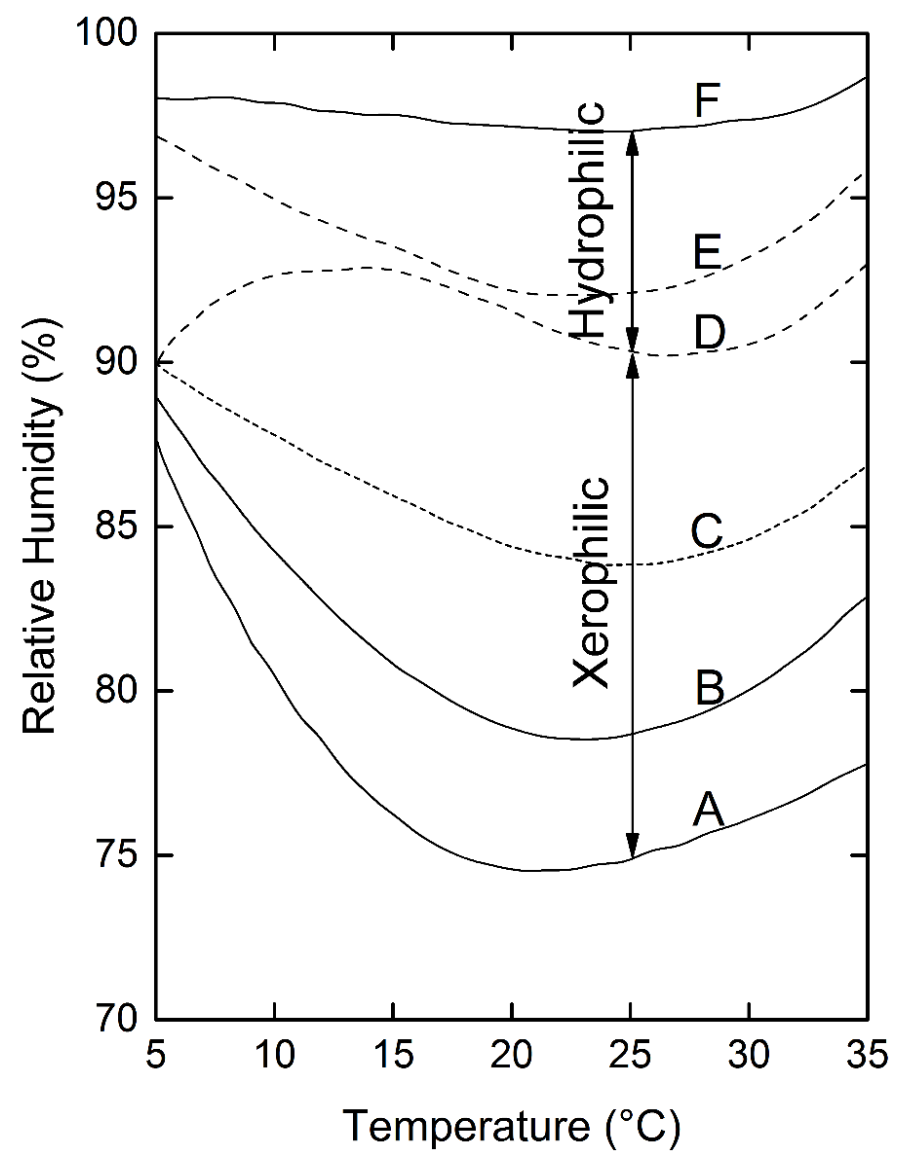

Fig. 1 Mould Growth Isopleth. Adapted from (Clarke et al. 1997, Clarke 2001)

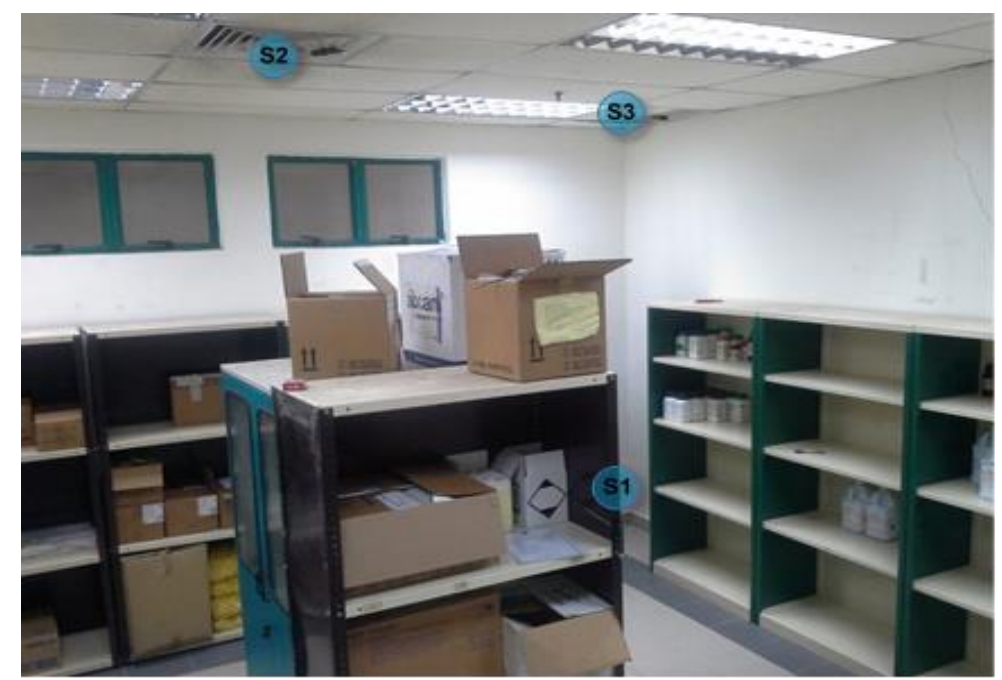


Fig. 2 Layout and measurement positions in the in-situ experimental setup

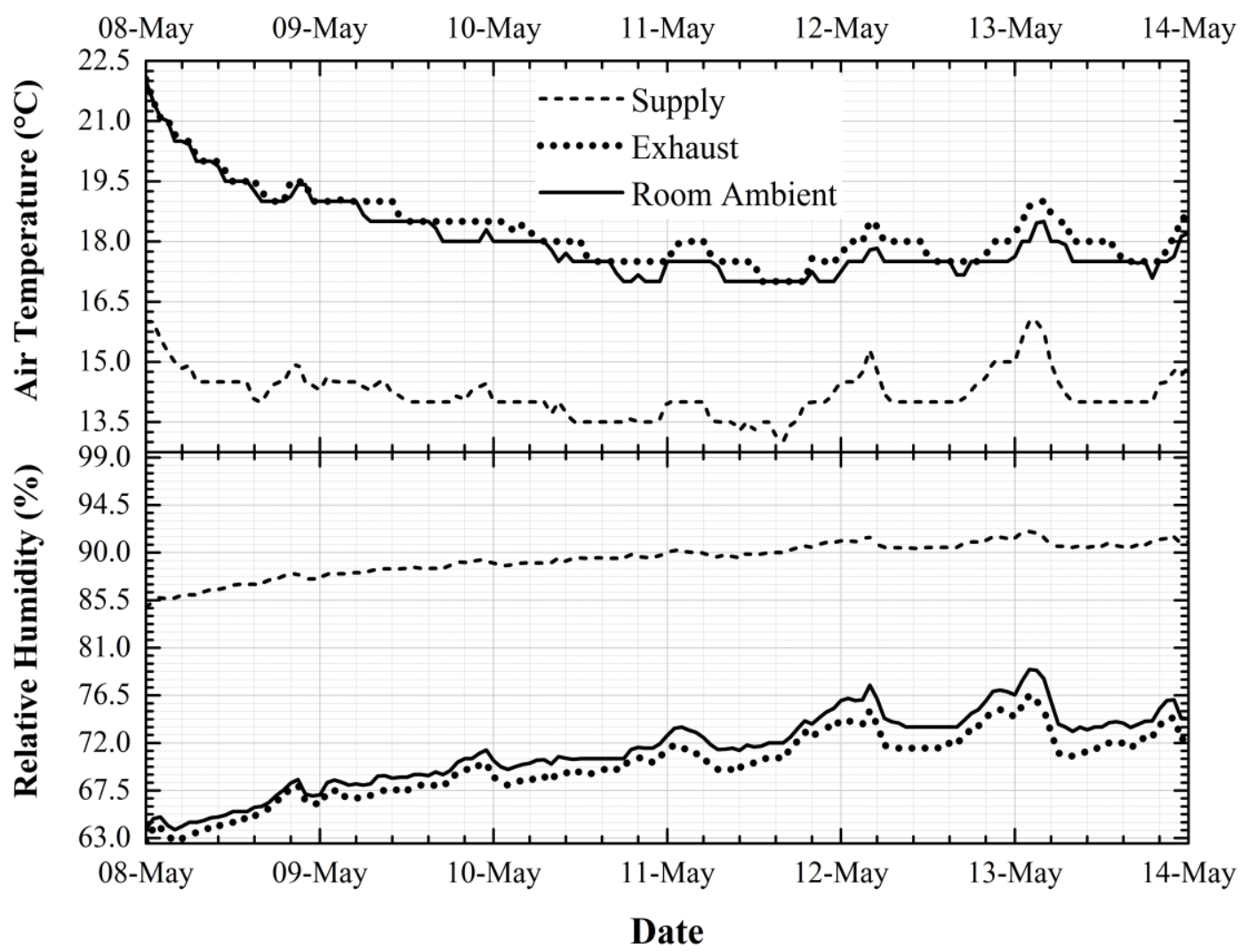

Fig. 3 Time-series Data of Hygrothermal Profile in the Case-studied Room 
Building Simulation

Volume 10, pages 551-562(2017)

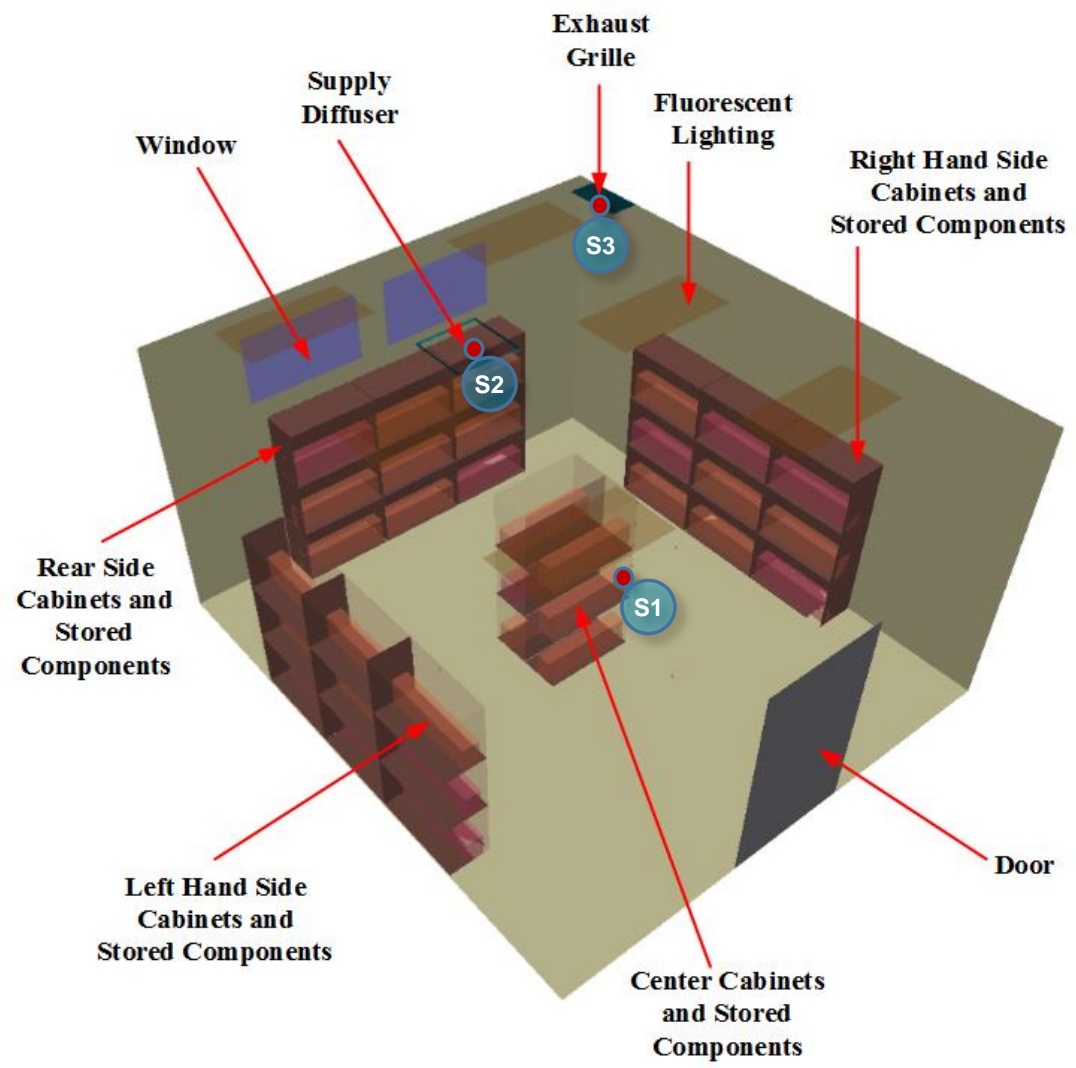

Fig. 4 Space Layouts with positions of supply, exhaust and other components of the case study room (S1, S2 and S3 are positions of virtual data loggers)
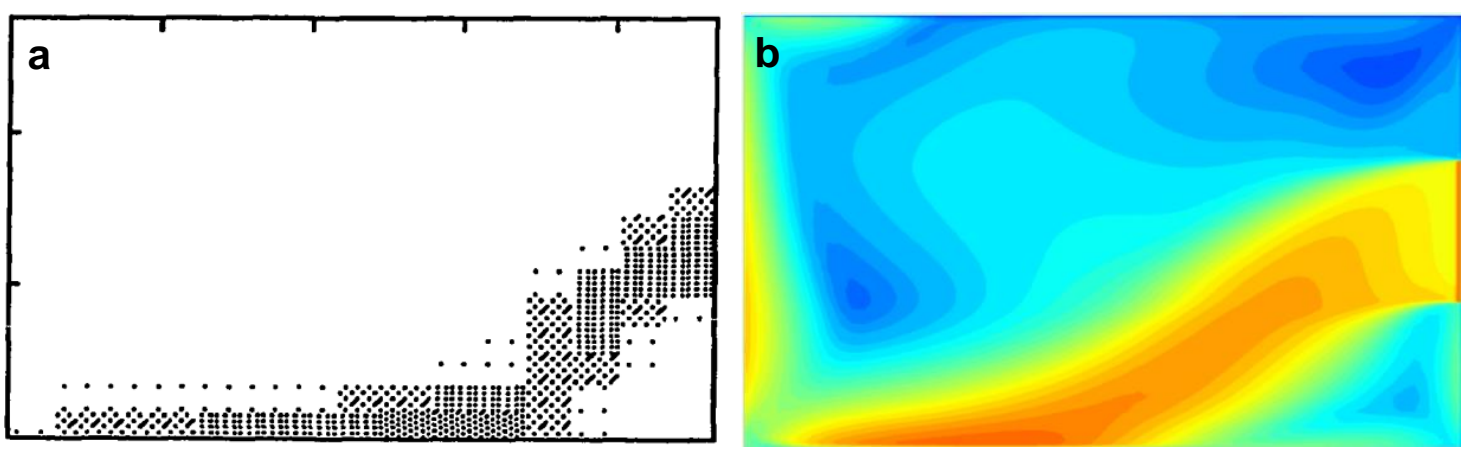

Fig. 5 Benchmark case: airflow profile with side mounted supply (a) Spitler (1990) experimental airflow pattern (b) CFD simulation airflow pattern 
Building Simulation

Volume 10, pages 551-562(2017)

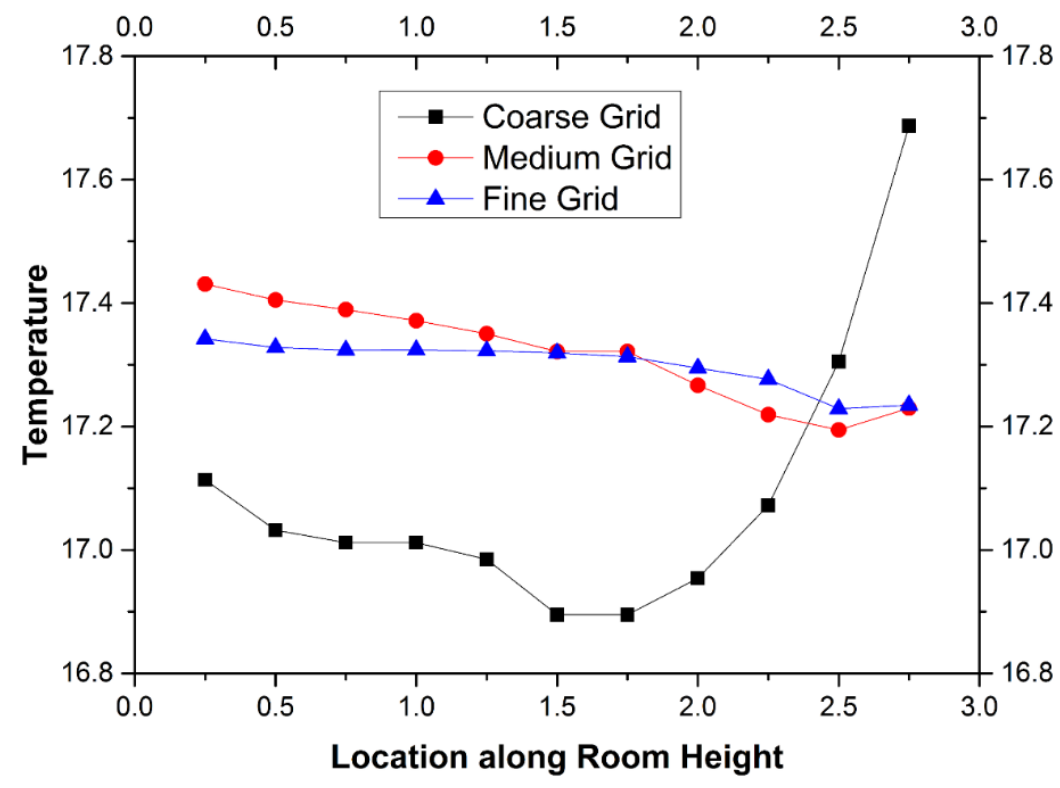

Fig. 6 Grid Dependency Analysis
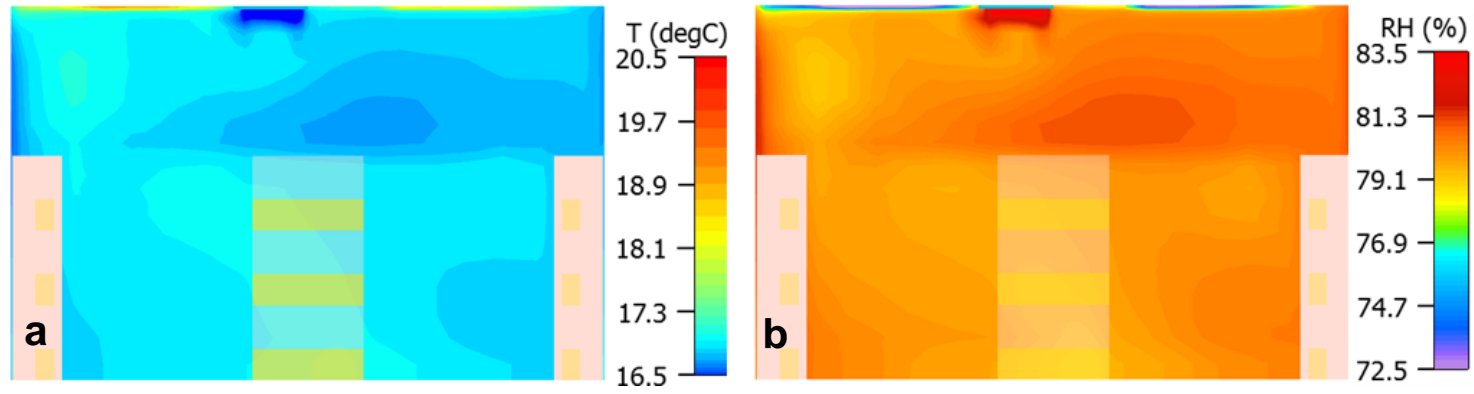

Fig. 7 Thermohygric profile at $x=0.75 \mathrm{~m}$ (a) T contour (b) RH contour
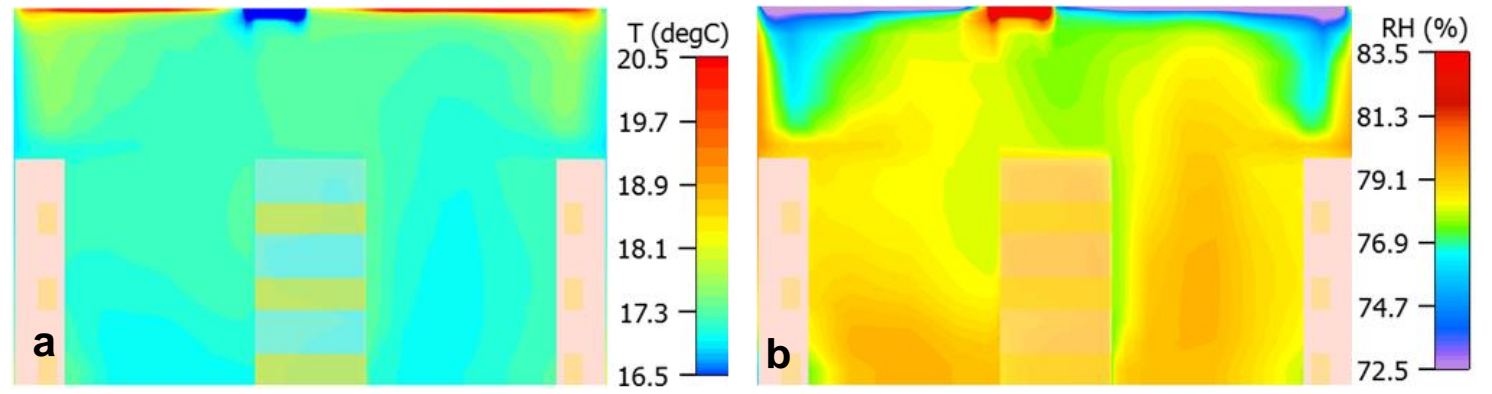

Fig. 8 Thermohygric profile at $x=2.25 \mathrm{~m}$ (a) T contour (b) RH contour 


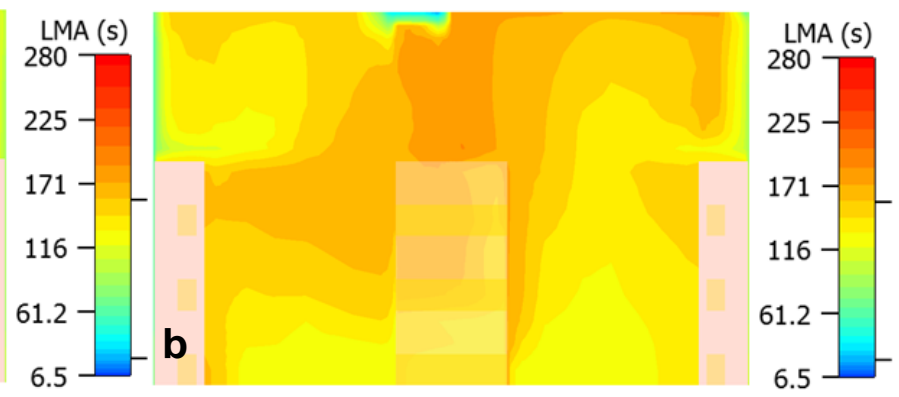

Fig. 9 LMA profile at (a) $x=0.75 \mathrm{~m}$ and (b) $x=2.25 \mathrm{~m}$

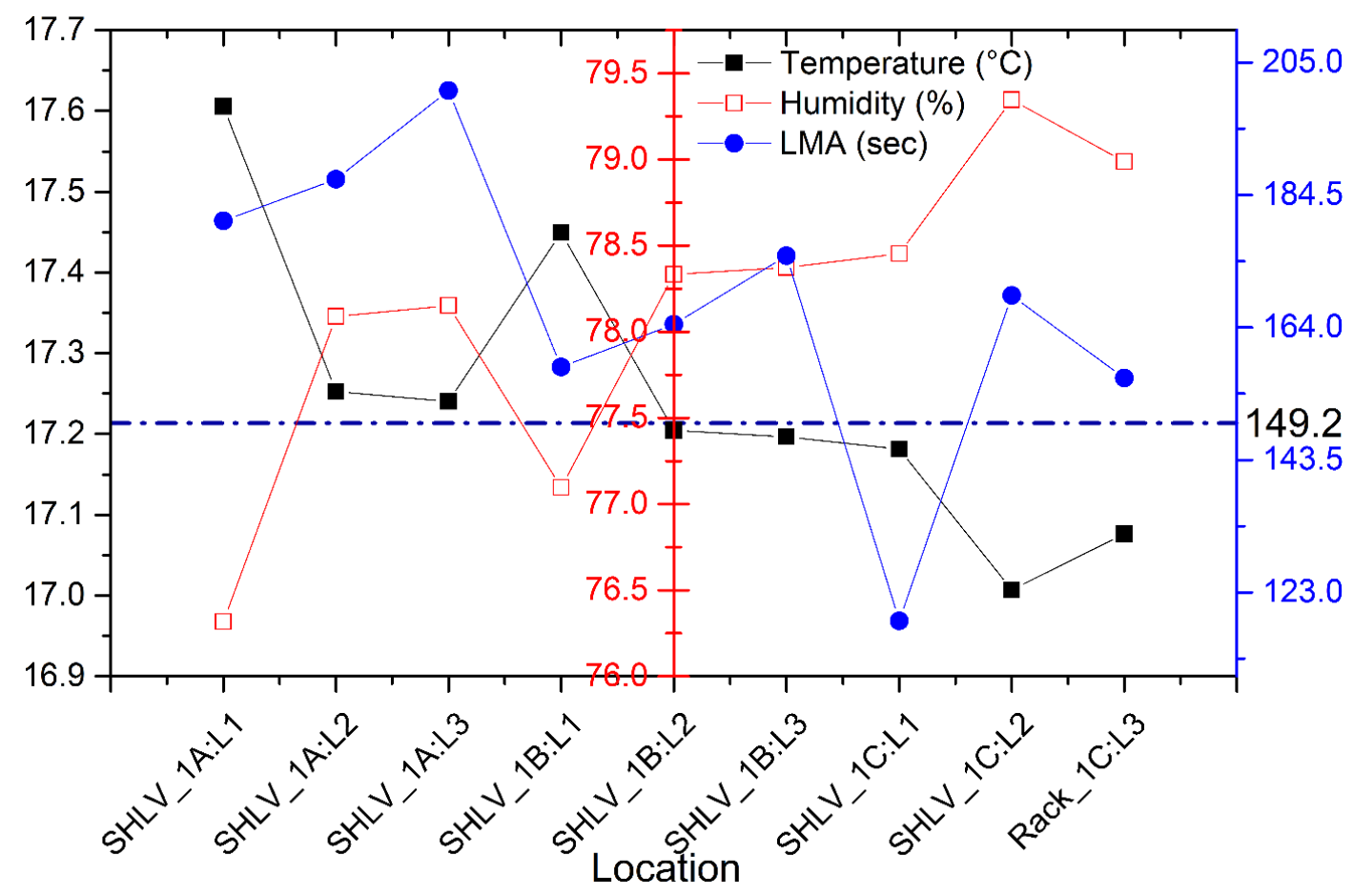

Fig. 10 Thermohygric and LMA profiles inside shelve \#1 


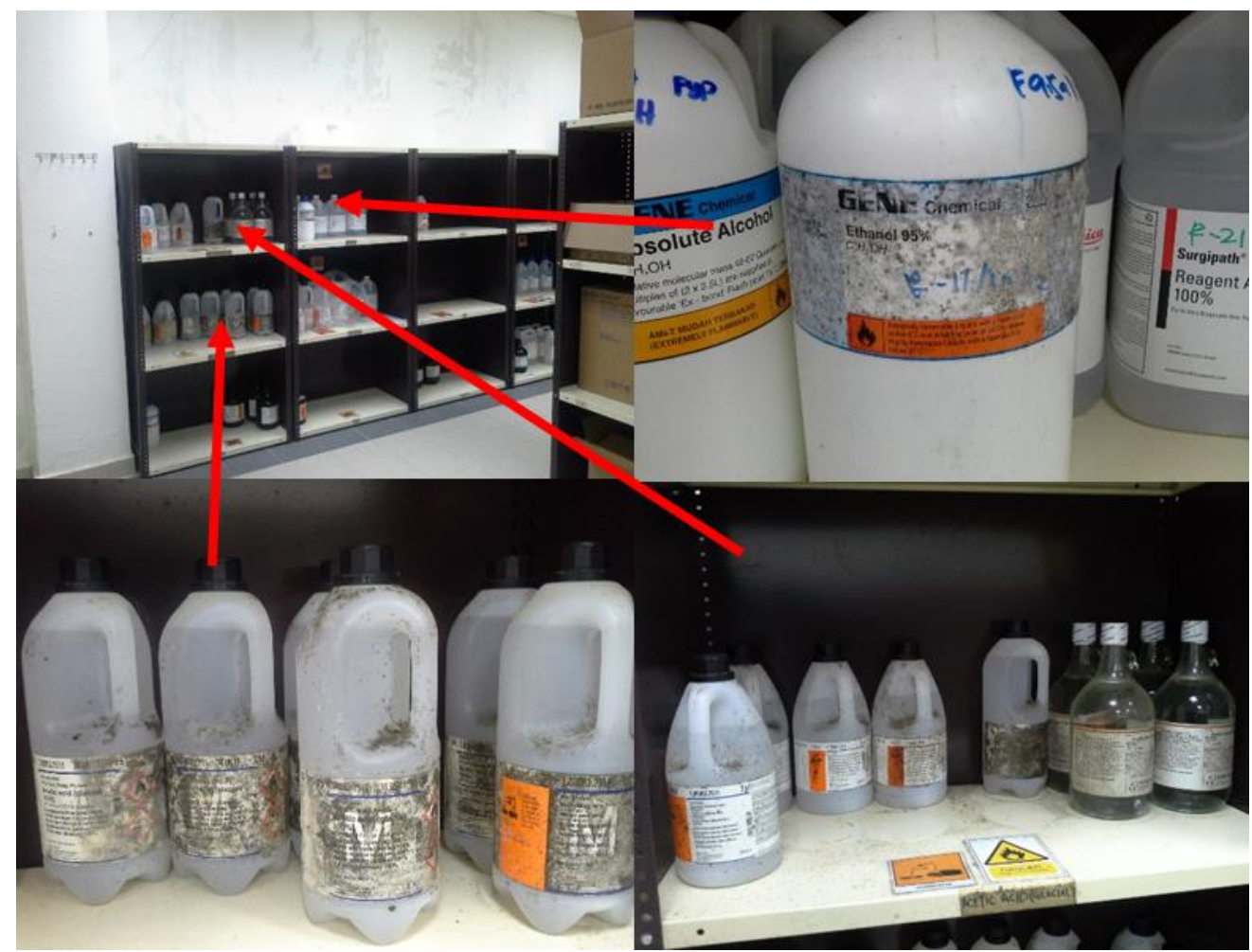

Fig. 11 Mould Growth on Stored items inside shelve \#1

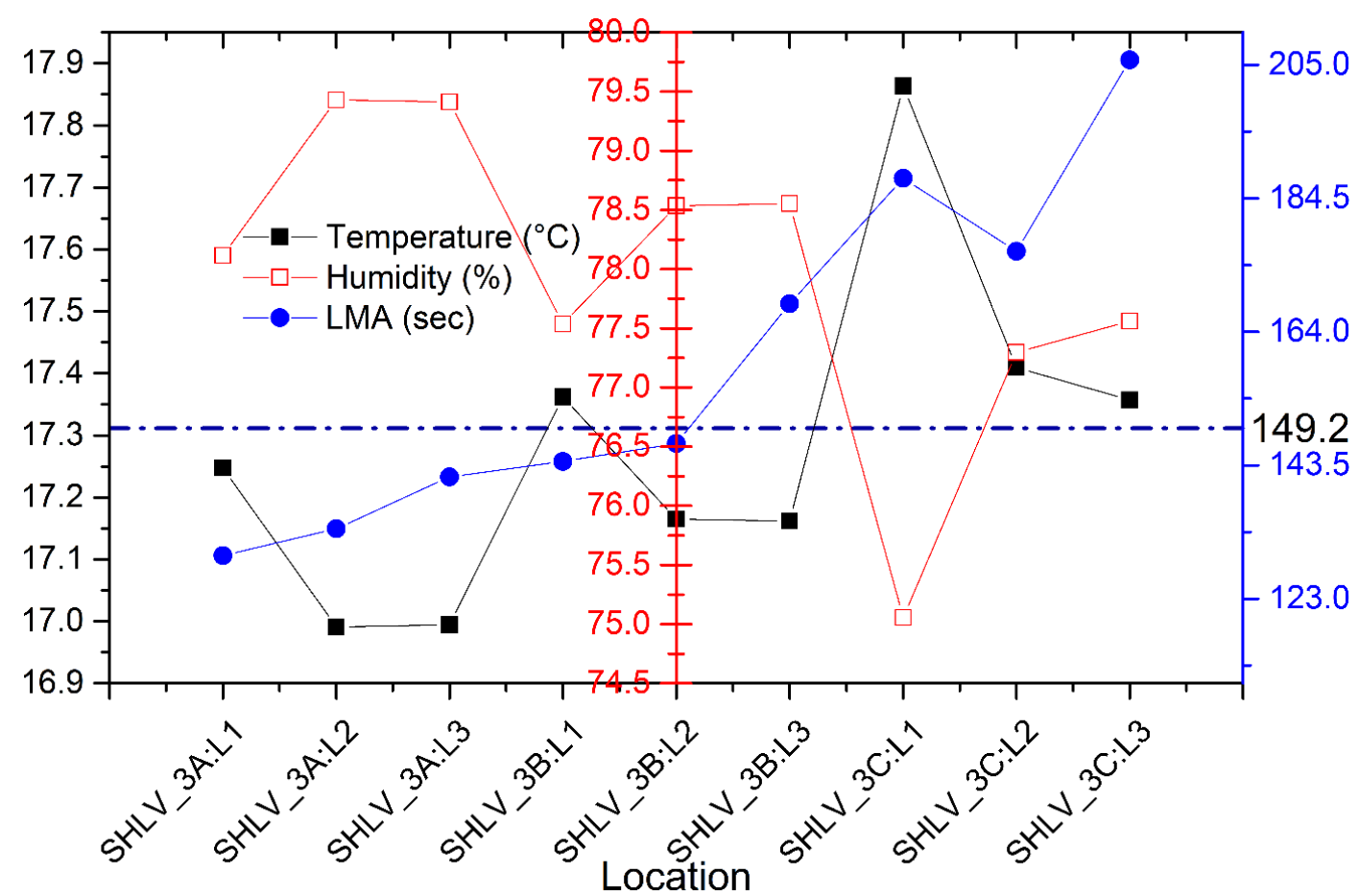

Fig. 12 Thermohygric and LMA profiles inside shelve \#3 


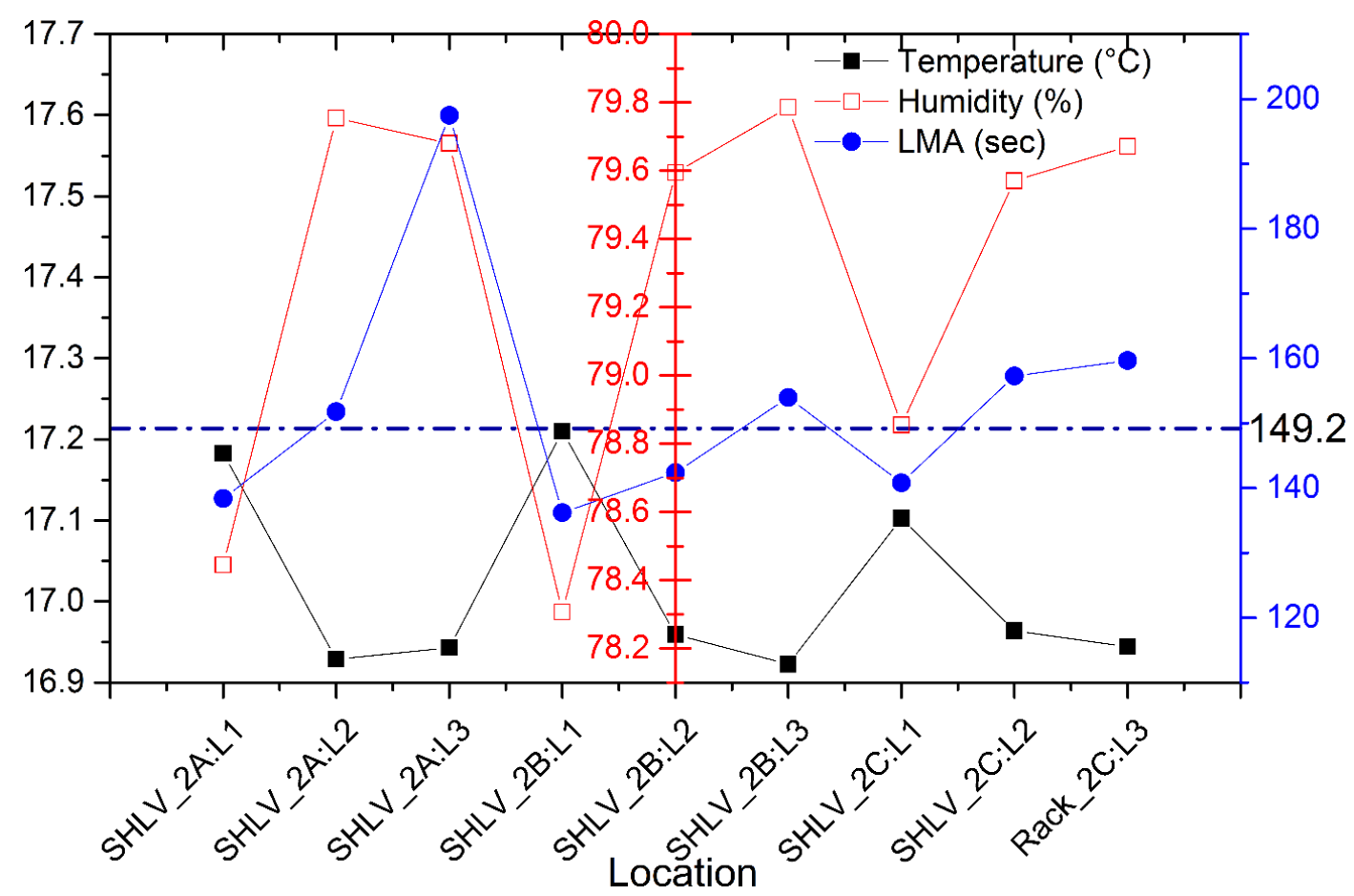

Fig. 13 Thermohygric and LMA profiles inside shelve \#2 

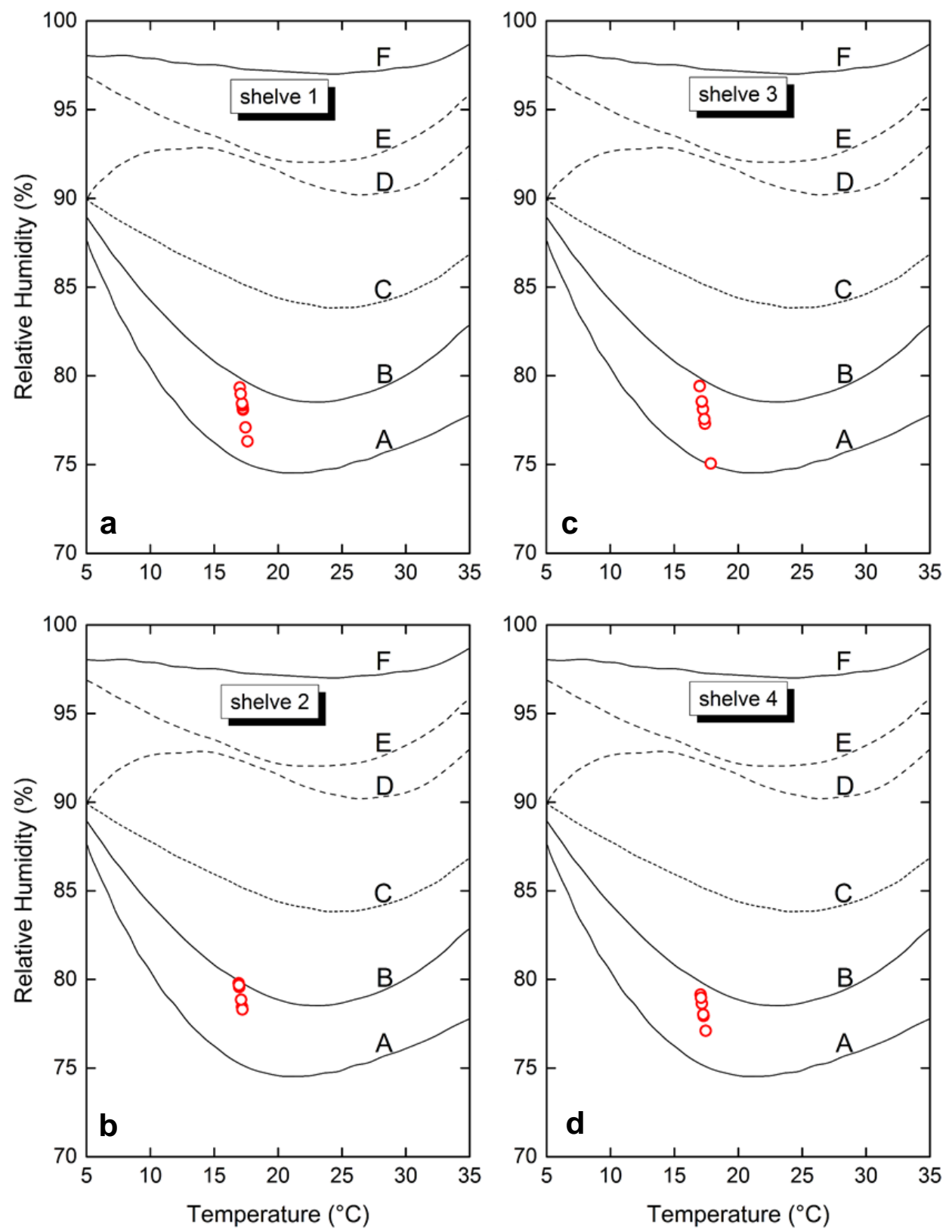

Fig. 14 Mould Growth Prediction on Stored Components in: (a) shelve \#1 (b) shelve \#2 (c) shelve \#3 and (d) shelve \# 4 


\section{List of Table}

Table 1 Specifications of the measurement equipment

\begin{tabular}{|c|c|c|c|c|c|}
\hline Equipment & Variable & Accuracy & Resolution & Range & Manufacturer \\
\hline \multirow{3}{*}{$\begin{array}{l}\text { ALNOR } \\
\text { AVM440 } \\
\text { Airflow } \\
\text { Instrument }\end{array}$} & RH & $\pm 3 \%$ & $0.1 \%$ & \begin{tabular}{|l|}
5 to \\
$05 \%$
\end{tabular} & \multirow{3}{*}{$\begin{array}{c}\text { TSI } \\
\text { Incorporation. }\end{array}$} \\
\hline & $\mathrm{T}$ & $\pm 0.3^{\circ} \mathrm{C}$ & $0.1^{\circ} \mathrm{C}$ & $\begin{array}{l}-10 \text { to } \\
60^{\circ} \mathrm{C}\end{array}$ & \\
\hline & V & $\begin{array}{l} \pm 3 \% \text { or } \\
\pm \\
0.015 \mathrm{~m} / \mathrm{s}\end{array}$ & $0.01 \mathrm{~m} / \mathrm{s}$ & $\begin{array}{l}0 \text { to } \\
30 \mathrm{~m} / \mathrm{s}\end{array}$ & \\
\hline \multirow{2}{*}{$\begin{array}{l}\text { EL-USB-2- } \\
\text { LCD+ Data } \\
\text { Logger } \\
\text { with LCD }\end{array}$} & RH & $\pm 3 \%$ & $0.5 \%$ & \begin{tabular}{|l|}
0 to \\
$100 \%$ \\
\end{tabular} & \multirow{2}{*}{$\begin{array}{l}\text { LASCAR } \\
\text { Electronics }\end{array}$} \\
\hline & $\mathrm{T}$ & $\pm 0.5^{\circ} \mathrm{C}$ & $0.5^{\circ} \mathrm{C}$ & $\begin{array}{l}-35 \text { to } \\
80^{\circ} \mathrm{C} \\
\end{array}$ & \\
\hline $\begin{array}{l}\text { Testo } \\
\text { Quicktemp } \\
\text { 860-T1 }\end{array}$ & $\mathrm{T}$ & $\begin{array}{l} \pm 0.75 \% \\
\text { or } \\
\pm 0.75^{\circ} \mathrm{C}\end{array}$ & $0.1^{\circ} \mathrm{C}$ & $\begin{array}{l}-30 \text { to } \\
900^{\circ} \mathrm{C}\end{array}$ & Testo AG \\
\hline
\end{tabular}

Table 2 The dependent variables $(\phi)$, effective diffusion coefficients $(\Gamma \phi)$, and the source term (S $\Phi)$ for each of the flow parameters

\begin{tabular}{|c|c|c|c|}
\hline Equation & $\Phi$ & $\Gamma_{\Phi}$ & $S_{\Phi}$ \\
\hline Continuity & 1 & 0 & 0 \\
\hline$x$ momentum & $u$ & $\mu$ & $-\partial P / \partial x$ \\
\hline $\begin{array}{l}y \text { momentum } \\
\text { (vertical) }\end{array}$ & $v$ & $\mu$ & $-\partial P / \partial y-\rho g$ \\
\hline$z$ momentum & $w$ & $\mu$ & $-\partial P / \partial z$ \\
\hline Enthalpy & $C_{p} T$ & $\lambda$ & $Q$ \\
\hline Concentration & $c / \rho$ & $d$ & $Q_{m}$ \\
\hline$k$ equation & $k$ & $\mu / \sigma_{k}$ & $G-\rho \varepsilon$ \\
\hline$\varepsilon$ equation & $\varepsilon$ & $\mu / \sigma_{\varepsilon}$ & $C_{1} \varepsilon G / k-C_{2} \rho \varepsilon_{2} / k$ \\
\hline \multicolumn{4}{|c|}{$\begin{array}{l}\mu=\mu_{\text {lam }}+\mu_{t} \\
\mu_{t}=\rho C \mu k^{2} / \varepsilon \\
\mathrm{G}=\mu\left[2\left[(\partial \mathrm{u} / \partial x)^{2}+(\partial \mathrm{v} / \partial y)^{2}+(\partial w / \partial z)^{2}\right]+(\partial u / \partial y+\partial v / \partial x)^{2}+(\partial v / \partial z+\partial w / \partial y)^{2}+(\partial u / \partial z\right. \\
\left.+\partial w / \partial x)^{2}\right] \\
\mathrm{C} 1=1.44, \mathrm{C} 2=1.92, \mathrm{C} \mu=0.09, \sigma_{H}=0.9, \sigma_{k}=1.0, \sigma_{\varepsilon}=1.3\end{array}$} \\
\hline
\end{tabular}


Table 3 Comparison of equipment uncertainty between measured and manufacturer specifications

\begin{tabular}{|l|l|l|l|l|}
\hline \multirow{2}{*}{ Sensors } & \multicolumn{2}{|l|}{ Measured Uncertainty } & \multicolumn{2}{l|}{ Manufacturer Specification } \\
\cline { 2 - 5 } & $\mathbf{T}\left({ }^{\circ} \mathbf{C}\right)$ & $\mathbf{R H}(\%)$ & $\mathbf{T}\left({ }^{\circ} \mathbf{C}\right)$ & RH $(\%)$ \\
\hline S1 & \pm 0.37 & \pm 0.72 & \pm 0.5 & \pm 3.0 \\
\hline S2 & \pm 0.11 & \pm 0.72 & \pm 0.5 & \pm 3.0 \\
\hline S3 & \pm 0.19 & \pm 0.63 & \pm 0.5 & \pm 3.0 \\
\hline$p<0.05$ & \multicolumn{4}{|l}{} \\
\hline
\end{tabular}

